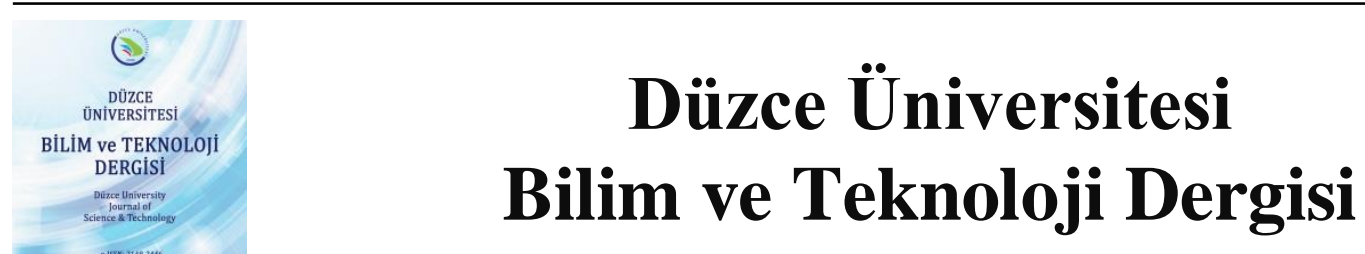

Araştırma Makalesi

\section{Tek Bölgeli Bir Güç Sisteminde Bulanık Mantık Tabanlı Yük Frekans Kontrolü (YFK)}

\author{
(D) Özay CAN ${ }^{\text {a,** }}$, (D) Ali ÖZTÜRK ${ }^{\text {b }}$ \\ ${ }^{a}$ Elektrik ve Enerji Bölümü, Kavak Meslek Yüksekokulu, Samsun Üniversitesi, Samsun, TÜRKIYE \\ ${ }^{b}$ Elektrik-Elektronik Mühendisliği Bölümü, Mühendislik Fakültesi, Düzce Üniversitesi, Düzce, TÜRKiYE \\ * Sorumlu yazarm e-posta adresi: ozay.can@ samsun.edu.tr
}

DOI : 10.29130/dubited.731579

\begin{abstract}
ÖZET
Bu çalışmada, tek bölgeli bir güç sisteminde yük frekans kontrolü (YFK) gerçekleştirilmiştir. YFK, sistemdeki ani yük değişimleri sonucu artan veya azalan frekansı nominal değerinde tutma işlemidir. Yapılan çalışmada, öncelikle tek bölgeli güç sistemi MATLAB/SIMULINK ortamında modellenmiştir. Daha sonra kontrolör olarak klasik PI kontrolör ve bulanık PI kontrolör kullanılarak frekans değişiminin sıfir olması amaçlanmıştır. Sisteme uygulanan yük değişimlerine karşı sistemin frekansında meydana gelen değişimler her iki kontrolör için elde edilmiştir. Bulanık PI kontrolör ve klasik PI kontrolör için elde edilen sonuçlar, bulanık mantık kontrolör ile yapılan YFK işleminde sistem frekansının nominal değerine daha kısa sürede ulaştığını ve sistemin aşma değerinin daha küçük olduğunu göstermiştir.
\end{abstract}

Anahtar Kelimeler: Yük frekans kontrolü (YFK), Bulanık mantık, Tek bölgeli güç sistemi.

\section{Fuzzy Logic Based Load Frequency Control (LFC) in a Single Area Power System}

\begin{abstract}
In this study, load frequency control (LFC) is performed in a single area power system. LFC is the process to keep the frequency that increases or decreases at its nominal value as a result of sudden load changes in the system. In the study, firstly, the single area power system was modeled in MATLAB/SIMULINK environment. Then, it was intended to be zero the frequency using the conventional PI controller and fuzzy PI controller as the controller. Changes in the frequency of the system against load changes applied to the system have obtained for both controllers. The results obtained for the fuzzy PI controller and the classical PI controller show that the system frequency has reached its nominal value in a shorter time and the overshoot value of the system is smaller in the LFC operation with fuzzy logic controller.
\end{abstract}

Keywords: Load frequency control (LFC), Fuzzy logic, Single area power system.

Geliṣ: 05/03/2020, Düzeltme: 04/11/2020, Kabul: 12/11/2020 


\section{GIRIS}

Büyük ölçekli enterkonnekte güç sistemlerinin çalışması ve kontrolünde, yük frekans kontrolü (YFK) veya otomatik üretim kontrolü (OÜK) konusu, tüketicilere güvenilir ve yüksek kaliteli elektrik enerjisi sağlamak açısından çok önemlidir. Güç sistemlerinde tüketicilerin talepleri nedeniyle meydana gelen ani yük değişimleri, güç sisteminin çalışma noktasının değişmesine, frekansın nominal değerinden sapmasına ve sistemin kararsızlığa gitmesine neden olmaktadır. YFK probleminin temel amacı, frekanstaki sapma ve güç sistemindeki yük taleplerinin daha iyi takip edilmesi için kararlı durum hatalarını en aza indirmektir.

Normal durumda, sistemdeki senkron generatörlerin güç çıkışı ve anlık yük değişimi arasındaki fark, nominal frekansı değiştirir. Talep edilen güç ile üretim arasında denge olmaması durumunda, frekans artar veya azalır. Güç sistemlerinde üretilen güç miktarı talep edilen güçten büyükse, generatörlerin çalışma hızı artar ve bu nedenle frekans artar. Benzer şekilde, sistemdeki üretim miktarı talep edilen güçten düşükse, sistem frekansı azalır.

YFK, primer frekans kontrolü, ve sekonder frekans kontrolü olmak üzere iki aşamadan oluşmaktadır. Tek bölgeli frekans kontrolüne primer frekans kontrolü de denir. Primer frekans kontrolü, yük değişimi sonucu artan veya azalan frekans değerinin en fazla 30 sn içinde -bu değer TEİAŞ yönetmeliklerinde verilmiştir- belirli sınırlar içinde $( \pm 200 \mathrm{mHz})$ tutulmasıdır. Bu süre içerisinde bu işlem gerçekleştirilimezse sekonder frekans kontrolü devreye girer. Bu da otomatik üretim kontrolü olarak adlandırılır. Sekonder frekans kontrolünde, primer frekans kontrolünde kontrol altına alınan frekansın nominal değerine getirilmesi sağlanır.

YFK ile ilgili literatür çalışması yapıldığında, bulanık mantık, yerçekimi arama algoritması (YAA), yapay sinir ağları (YSA), optikten esinlenen optimizasyon algoritması, parçacık sürü optimizasyonu (PSO), genetik algoritma (GA), klasik PID kontrolör, HVDC FACTS kontrolör ve kesir dereceli kontrolör gibi yöntemlerin kullanıldığı görülmektedir.

Tek bölgeli veya çok bölgeli güç sisteminin YFK işlemi için bulanık mantık yönteminin kullanıldığ çalışmalarda, elde edilen sonuçlar klasik kontrolör kullanılarak yapılan YFK sonuçları ile kıyaslanmıştır. Elde edilen sonuçlar, bulanık mantık yönteminin daha iyi performans sergilediğini göstermiştir [1-11]. Yerçekimi arama algoritması (YAA) yönteminin kullanıldığı çalışmalarda ise klasik PID parametreleri bu yöntemle optimize edilerek YFK işlemi gerçekleştirilmiştir [12-15]. Yapay sinir ağları (YSA) kullanarak gerçekleştirilen YFK işlemlerinde, kullanılan yöntemin klasik integral veya bulanık mantık yöntemine göre daha iyi sonuçlar verdiği gözlemlenmiştir [16-20]. YAA'ya benzer olarak PID parametrelerinin optimizasyonunda farklı teknikler kullanılarak YFK gerçekleştirilmiştir [21-23]. Bunun yanı sıra, farklı yöntemler kullanılarak da YFK işlemi uygulanabilir [24-26].

Gerçekleştirilen çalı̧̧mada ise bulanık mantık yöntemi ve klasik PI kontrolör yöntemi kullanılarak farklı yük değişimleri için YFK gerçekleştirilmiş olup sonuçlar karşılaştırılmıştır. Elde edilen sonuçlardan, kullanılan bulanık mantık yönteminin benzeri çalışmalarla kıyaslandığında \pm 0.01 pu'lik yük değişimindeki sistem frekansının aşma değeri bakımından [1,2,9,11]'deki değerlere göre, sistem frekasının oturma süresi bakımından [1,9]'daki değerlere göre daha iyi sonuçlar verdiği görülmüştür. Ayrıca yapılan benzer çalışmalardan farklı olarak, \pm 0.01 pu'lik yük değişiminin yanı sıra farklı yük 
değişimleri için de $( \pm 0.03 \mathrm{pu}$ ve $\pm 0.05 \mathrm{pu})$ bulanık mantık kontrolünün sistem frekansını istenen değerler arasında $( \pm 200 \mathrm{mHz})$ tutma noktasında başarılı olduğu gözlemlenmiştir.

\section{TEK BÖLGELİ GÜC SISTEMINDE YFK}

Tek bölgeli bir güç sisteminde, bir generatör ve bir türbin bulunmaktadır. Generatör ünitesinde mekanik güç türbin tarafindan üretilir ve elektrik gücünü üreten ve yüke sağlayan senkron generatöre iletilir. Gerilim ve frekanstaki sapmalar tüketiciye ulaştırılan gücün kalitesini etkiler. Bu kalite, türbin buhar akışı ve ayrıca yük/talep değişikliği ile sağlanır. Generatör üzerindeki yük artar veya azalırsa, generatörün mili yavaşlar veya hızlanır, bu da frekansın artmasını veya azalmasını etkiler. Değişimleri kontrol etmek için değişimi tespit eden ve buhar/su (basınç) vanasını açma veya kapama komutu veren kontrolörler gereklidir. Tek bölgeli bir güç sisteminin YFK blok diyagramı Şekil 1'de gösterilmiştir.

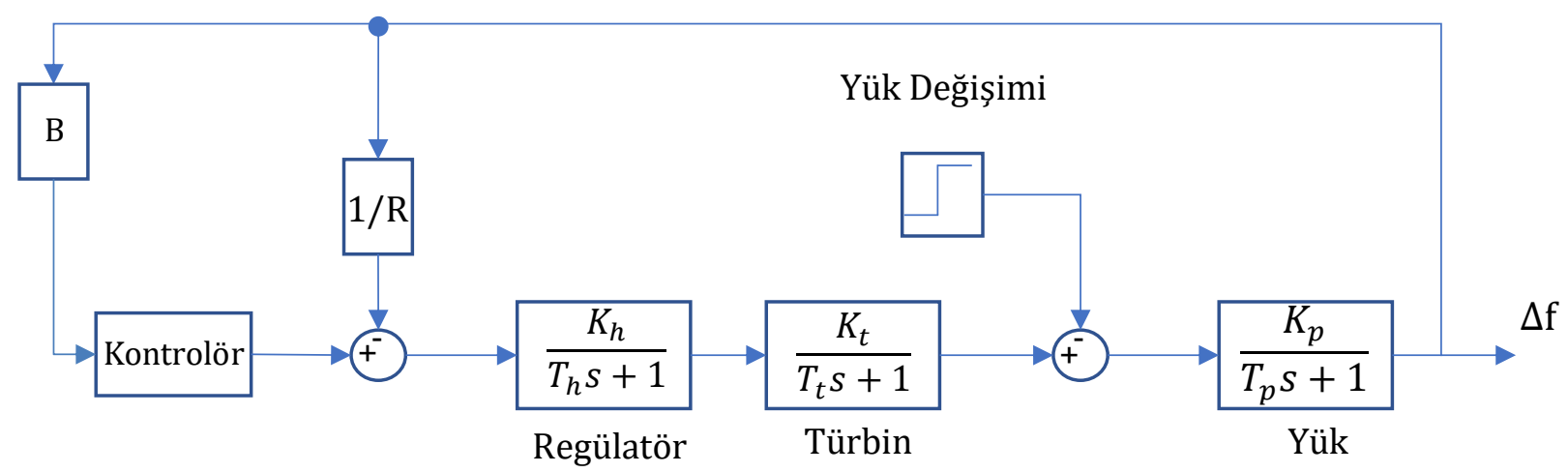

Şekil 1. Tek bölgeli sistem için YFK blok diyagramı [8].

Güç sistemlerinde, üretilen güç ile talep edilen güç arasında dengesizlik olması durumunda frekansın nominal değerinde tutulması gerekmektedir. Bu frekans değeri regülatörlere bağlıdır. Bu yüzden, YFK gerçekleştirilirken generatör ve türbinleri içeren sistem tümüyle hesaba katılmalıdır. Bu çalışmadaki sistem, türbin, generatör, kontrolör ve yük gibi birçok farklı bölümlerden oluşmaktadır.

Modelleme gerçekleştirilirken kullanılacak generatör ve türbinlerin transfer fonksiyonları Eşt. 1, Eşt. 2 ve Eşt. 3'te verilmiştir. Sistemdeki regülatörün transfer fonksiyonu Eşt. 1'de, türbinin transfer fonksiyonu Eşt. 2'de ve yükün transfer fonksiyonu Eşt. 3'te belirtilmiştir.

$T_{h}(s)=\frac{K_{h}}{T_{h} s+1}$

$T_{t}(s)=\frac{K_{t}}{T_{t} s+1}$

$T_{p}(s)=\frac{K_{p}}{T_{p} s+1}$

YFK işleminin gerçekleştirileceği sistemin paramatreleri Tablo 1'de gösterilmiştir. Tabloda, $\mathrm{R}$ regülasyon parametresini, $\mathrm{B}$ ise frekans sapmas1 parametresini gösterir. $T_{h}, T_{t}$ ve $T_{p}$ sirasiyla regülatörün, türbinin ve sistem yükünün zaman sabitelerini temsil etmektedir. $K_{h}, K_{t}$ ve $K_{p}$ ise sırasıyla regülatörün, türbinin ve sistem yükünün kazançlarını ifade etmektedir. 
Tablo 1. Sistem parametreleri.

\begin{tabular}{cc}
\hline Parametre & Değer \\
\hline$T_{h}$ & $0.08 \mathrm{sn}$ \\
\hline$T_{t}$ & $0.3 \mathrm{sn}$ \\
\hline$T_{p}$ & $20 \mathrm{sn}$ \\
\hline$K_{p}$ & $120 \mathrm{~Hz} / \mathrm{MW}$ \\
\hline $\mathrm{R}$ & $2.43 \mathrm{~Hz} / \mathrm{MW}$ \\
\hline $\mathrm{B}$ & $0.425 \mathrm{MW} / \mathrm{Hz}$ \\
\hline
\end{tabular}

Tek bölgeli güç sistemlerinde YFK gerçekleştirilirken farklı kontrolör yöntemleri kullanılabilir. Gerçekleştirilen çalışmada, klasik PI ve parametreleri bulanık mantık kontrolör tarafından belirlenen bulanık PI kontrolör kullanılmıştır. Sisteme uygulanan farklı yük değişimlerine göre sistem frekansının değişimini takip etmek ve kontrol etmek amacıyla bilgisayar ortamında tek bölgeli güç sisteminin simülasyon modeli oluşturulmuştur. Simülasyon için MATLAB programının SIMULINK arayüzü kullanılmıştır. Tek bölgeli güç sisteminin MATLAB/SIMULINK ortamında klasik PI kontrolör kullanılarak gerçekleştirilen simülasyon modeli Şekil 2'de verilmiştir.

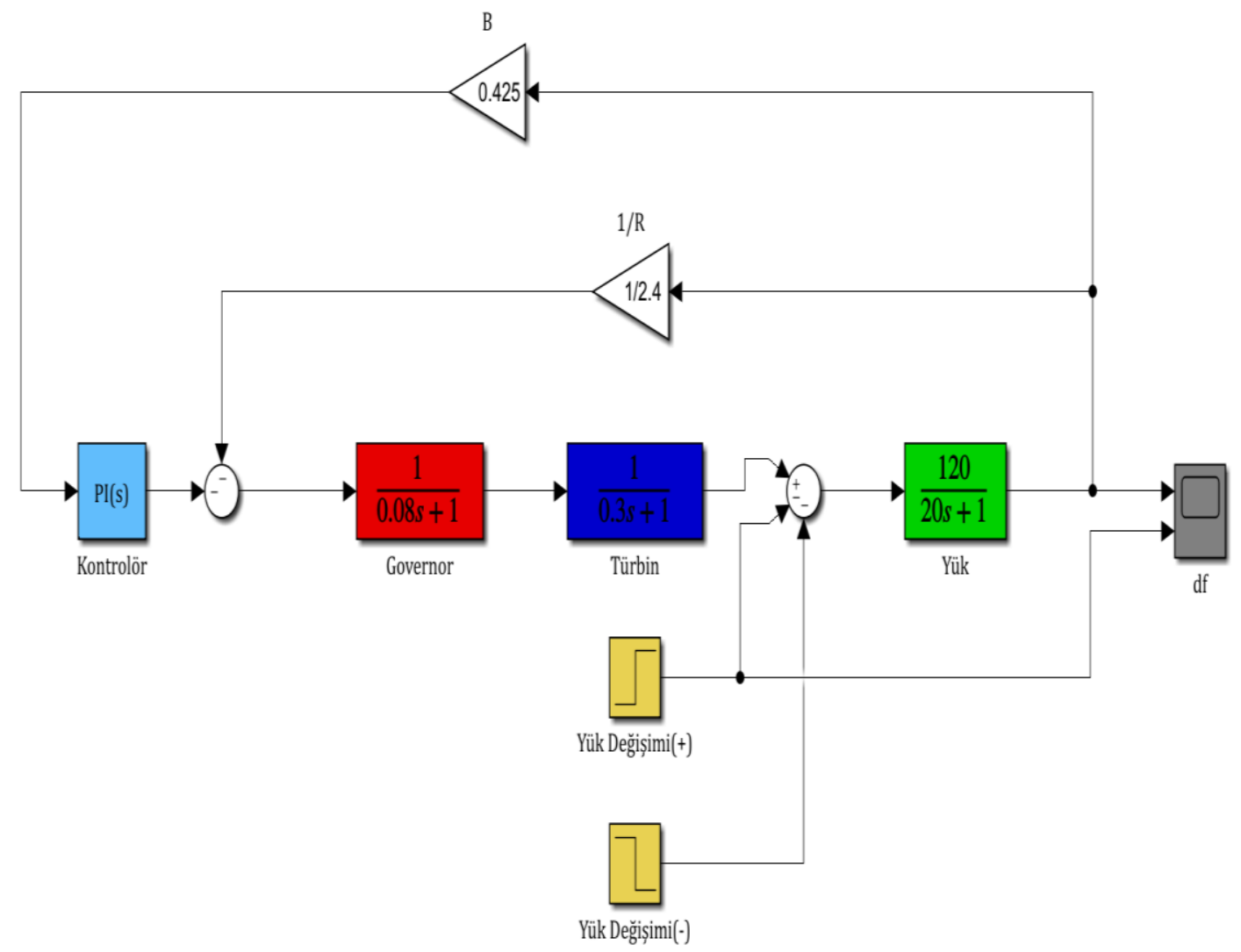

Şekil 2. Klasik PI kullanılarak gerçekleştirilen YFK modeli. 


\section{A. BULANIK MANTIK KONTROLÖR}

Güç sistemlerinin çok değişkenli koşullarından dolayı, geleneksel kontrol yöntemleri YFK problemleri için tatmin edici çözümler vermeyebilir. Bulanık sistemler, daha iyi sonuçlar vermesi amacıyla YFK problemine başarıyla uygulanabilir. Bulanık sistem, insan bilgisini matematiksel bir formüle dönüştürür. Bu nedenle, son yıllarda güç sistemi problemlerini çözmek için matematiksel yaklaşımlara tamamlayıcı bir araç olarak bulanık küme teorisine dayalı bir yaklaşım ortaya çıkmıştır.

Gerçekleştirilen YFK işleminde kontrolör olarak bulanık mantık kontrolör kullanılmıştır. Sisteme uygulanan yük değişimlerinde, sistem frekansında meydana gelen değişimlere bağlı olarak PI kontrolörün parametreleri belirlenir. Tek bölgeli güç sisteminin MATLAB/SIMULINK ortamında bulanık PI kontrolör kullanılarak gerçekleştirilen simülasyon modeli Şekil 3’te verilmiştir.

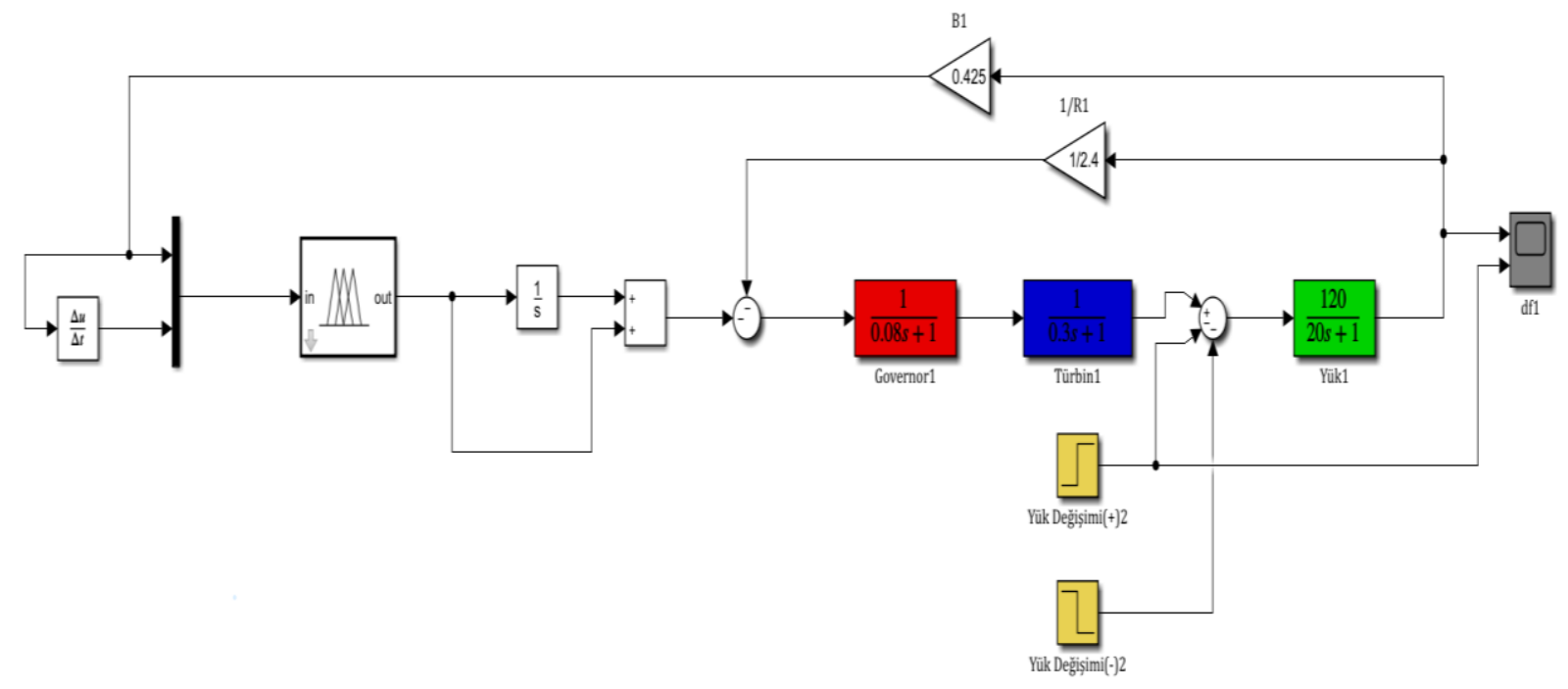

Şekil 3. Bulanık PI kullanılarak gerçekleștirilen YFK modeli.

Sistemde giriş olarak, sistem frekansının anlık değerinin nominal değer ile arasındaki hata ve bu hatanın değişimi, çıkış olarak ise kontrolör kazancı kullanılır. Şekil 4 'te ve Şekil 5'te sırasıyla hatanın ve hatanın değişiminin üyelik fonksiyonları verilmiştir.

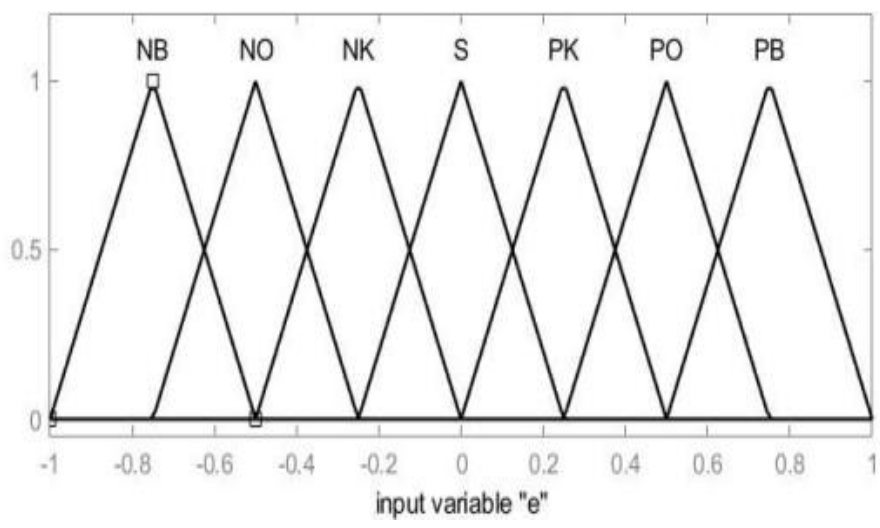

Şekil 4. Hatanın üyelik fonksiyonu. 


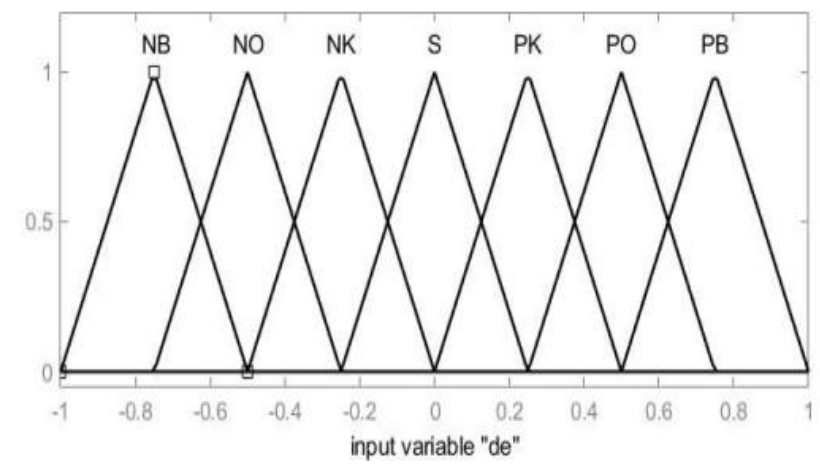

Şekil 5. Hatanın değişiminin üyelik fonksiyonu.

Çıkışın üyelik fonksiyonu ise Şekil 6' da gösterilmiştir.

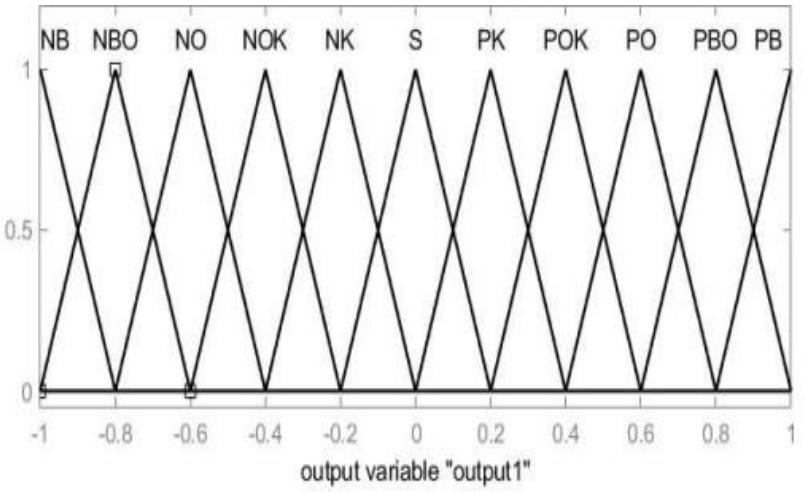

Şekil 6. Çıkışın üyelik fonksiyonu.

Girişlere göre çıkışın elde edilmesinde kullanılacak 7x7 kural tablosu Tablo 2'de verilmiştir.

Tablo 2. Kural tablosu.

\begin{tabular}{|c|c|c|c|c|c|c|c|}
\hline de & NB & NO & NK & $\mathbf{S}$ & PK & PO & PB \\
\hline NB & $N B$ & $N B$ & $N B O$ & $N O$ & NOK & $N K$ & $S$ \\
\hline NO & $N B$ & $N B O$ & $N O$ & $\mathrm{NOK}$ & $N K$ & $S$ & $P K$ \\
\hline NK & $N B O$ & $N O$ & NOK & $N K$ & $S$ & $P K$ & $P O K$ \\
\hline $\mathbf{S}$ & $N O$ & NOK & $N K$ & $S$ & $P K$ & $P O K$ & $P O$ \\
\hline PK & NOK & $N K$ & $S$ & $P K$ & $P O K$ & $P O$ & $P B O$ \\
\hline PO & $N K$ & $S$ & $P K$ & $P O K$ & $P O$ & $P B O$ & $P B$ \\
\hline PB & $S$ & $P K$ & $P O K$ & $P O$ & $P B O$ & $P B$ & $P B$ \\
\hline
\end{tabular}

NB: Negatif Büyük, NBO: Negatif Büyük Orta, NOK: Negatif Orta Küçük, NO: Negatif Orta, NK: Negatif Kü̧̈ük, S: Sifir, PK: Pozitif Küçük, PO: Pozitif Orta, POK: Pozitif Orta Küçük, PBO: Pozitif Büyük Orta, PB: Pozitif Büyük 


\section{BULGULAR}

Tek bölgeli bir güç sisteminde YFK işlemi gerçekleştirmek için ilk olarak MATLAB/SIMULINK ortamında sistemin simülasyon modeli oluşturulmuştur. Daha sonra, sisteme farklı zaman dilimlerinde farklı yük değişimleri ( $\pm 0.01 \mathrm{pu}, \pm 0.03 \mathrm{pu}, \pm 0.05 \mathrm{pu}$ ) uygulanarak sistem frekansının değişimleri ve kullanılan kontrolör yöntemlerinin sistem frekansı üzerindeki etkileri gözlemlenmiştir. Elde edilen sonuçlar grafiklerle verilmiştir.

Şekil 7'de sisteme uygulanan 0.01 pu'lik ve -0.01 pu'lik yük değişimleri verilmiştir. Sisteme t=5 sn anında 0.01 pu'lik, $\mathrm{t}=28$ sn anında -0.01 pu'lik yük değişimi uygulanmıştır.

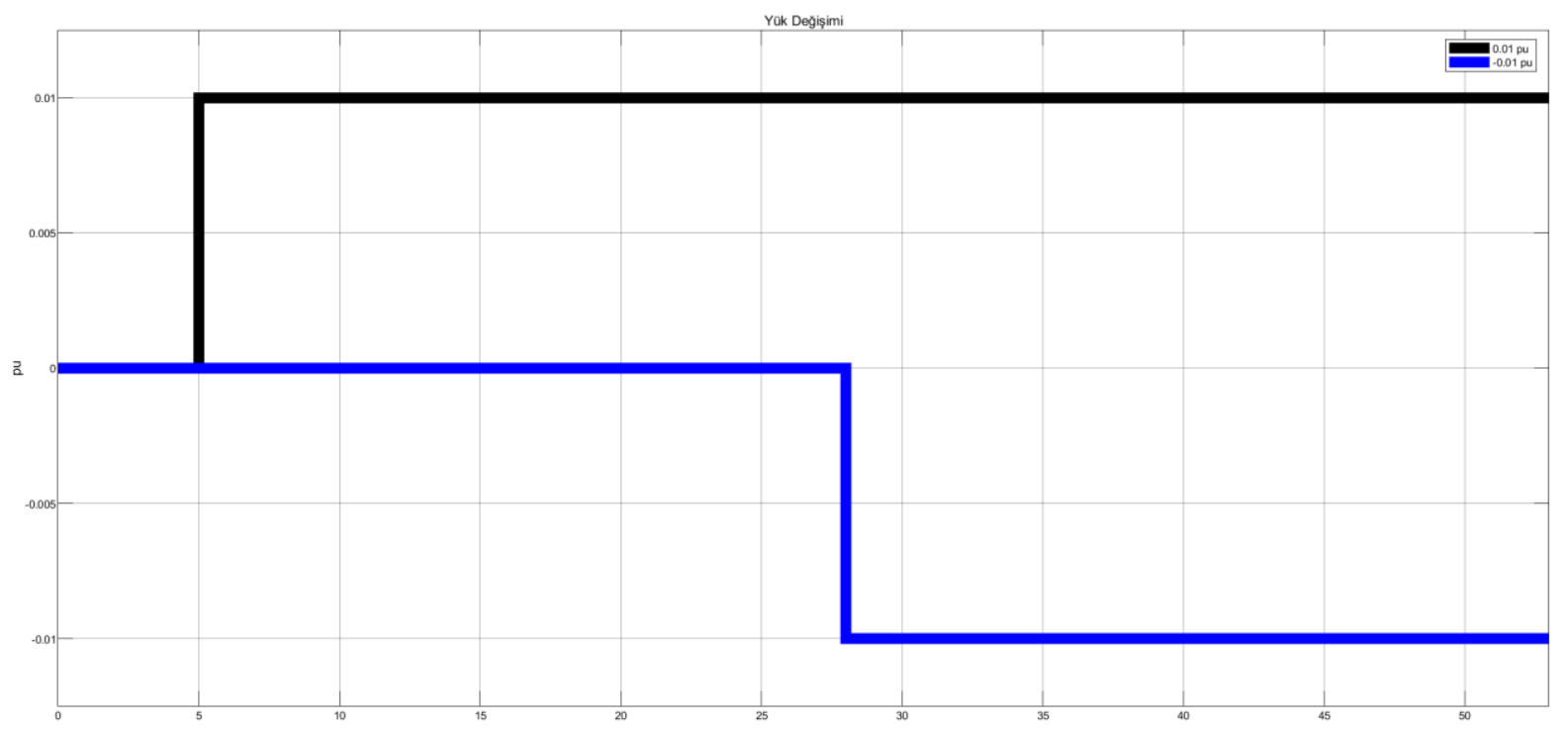

Şekil 7. $t=5$ sn için 0.01 pu'lik ve $t=28$ sn için -0.01 pu'lik yük değişimi.

Şekil 8'de ise bu yük değişimlerine karşı sistemdeki frekans değişimi her iki kontrol sistemi için gösterilmiştir. Şekilden görüldügüü üzere, 0.01 pu'lik yük değişimi için, sistemin maksimum salınım (aşma) değeri bulanık PI kontrolörü için yaklaşık 0.008 pu iken klasik PI kontrolörü için 0.028 pu civarındadır. Ayrıca, sistem frekansının değişiminin bulanık PI kontrolörü için 9 sn içinde, klasik PI kontrolü için ise $13 \mathrm{sn}$ içinde bastırıldığı görülmektedir.

Sisteme t=28 sn anında -0.01 pu'lik yük değişimi uygulandığında, 0.01 pu'lik yük değişimine benzer olarak, sistemin maksimum salınım (aşma) değeri bulanık PI kontrolörü için yaklaşık 0.008 pu iken klasik PI kontrolörü için 0.028 pu civarındadır. Ayrıca, sistem frekansının değişiminin bulanık PI kontrolörü için $\mathrm{t}=37$ sn anında, klasik PI kontrolü için ise $\mathrm{t}=41$ sn anında bastırıldığı görülmektedir. 


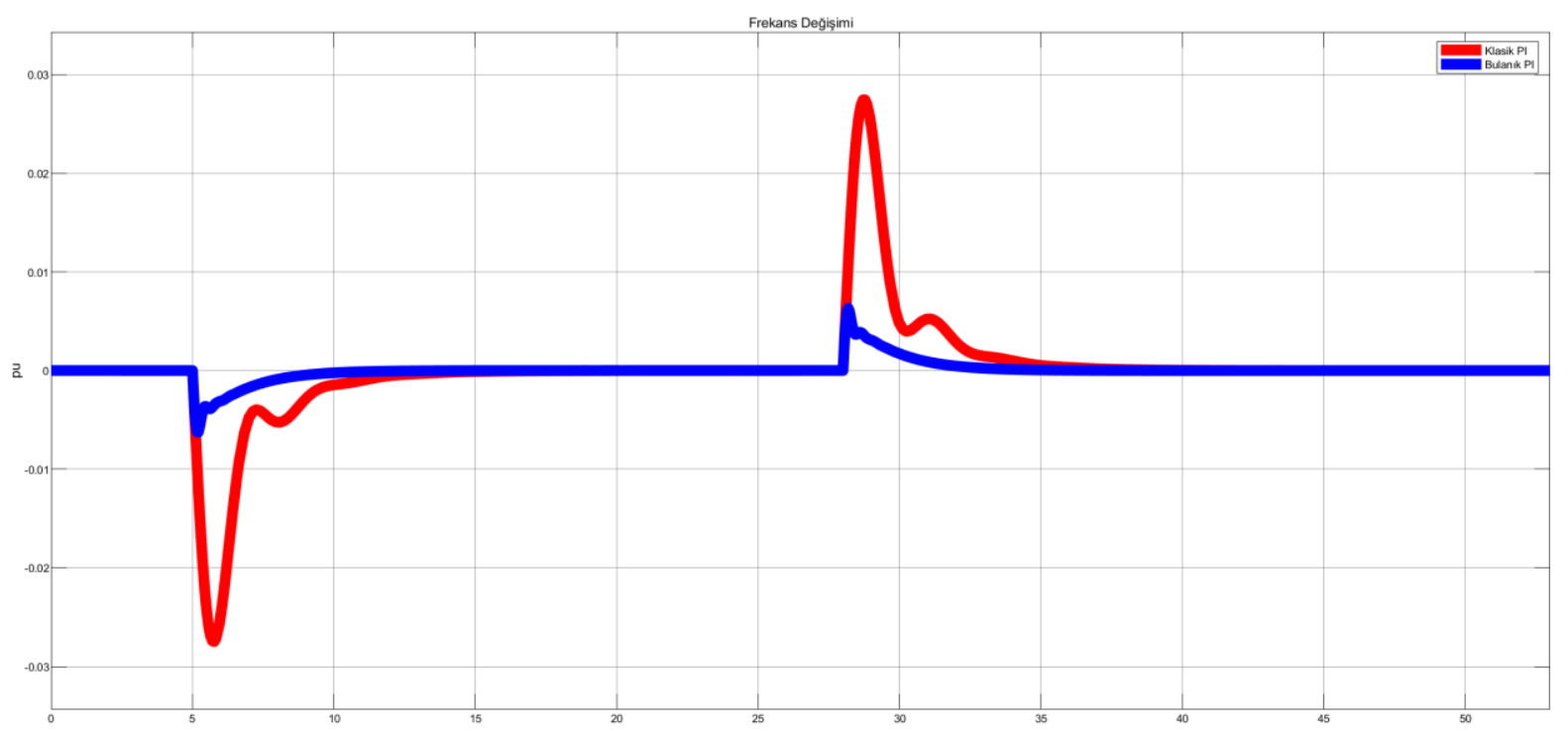

Şekil 8. 0.01 pu'lik ve -0.01 pu'lik yük değişimleri için frekans değişimi.

Şekil 9'da sisteme uygulanan 0.03 pu'lik ve -0.03 pu'lik yük değişimleri verilmiştir. Sisteme $t=5$ sn anında 0.03 pu'lik, t=28 sn anında -0.03 pu'lik yük değişimi uygulanmıştır.

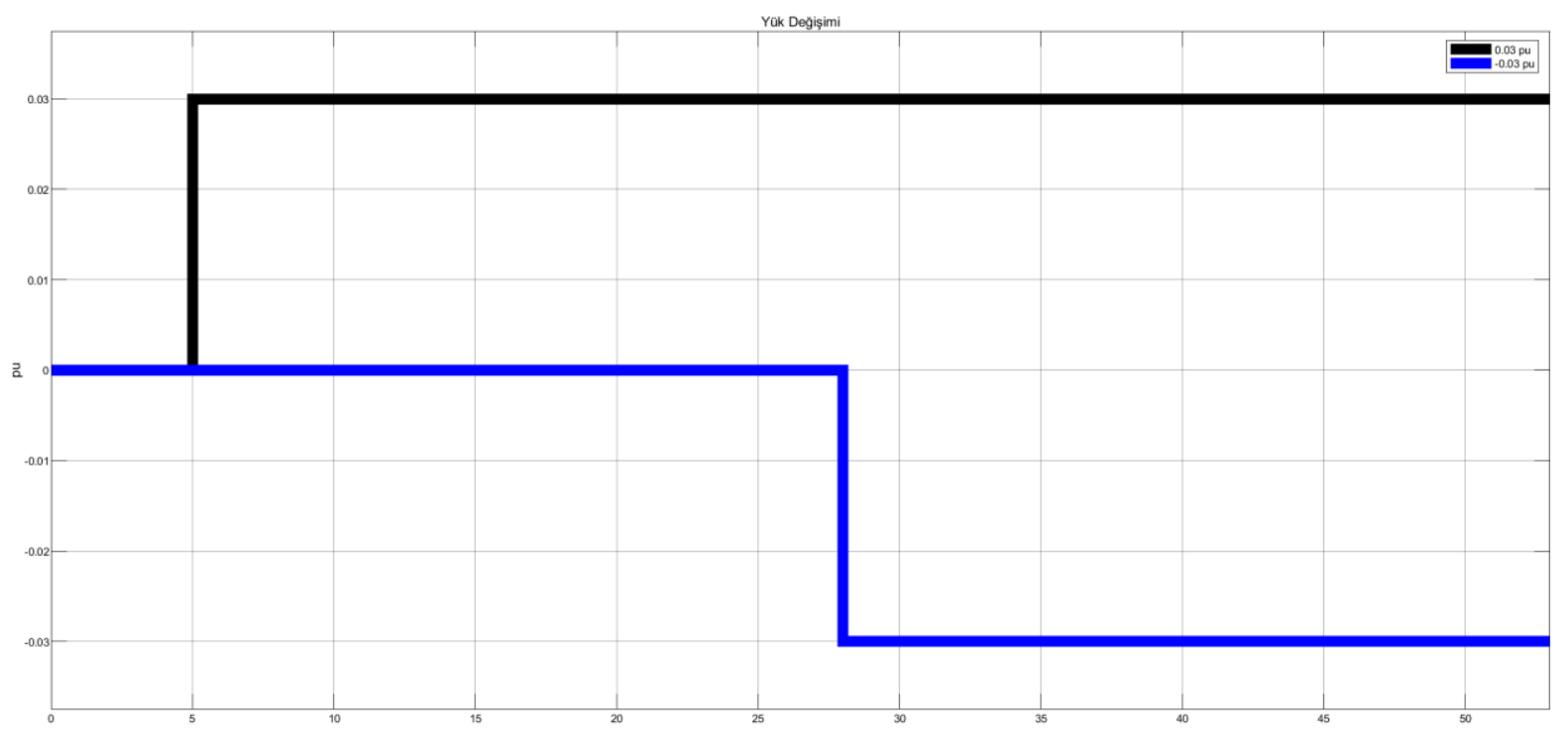

Şekil 9. $t=5$ sn için 0.03 pu'lik ve $t=28$ sn için -0.03 pu'lik yük değişimi.

Şekil 10'da ise bu yük değişimlerine karşı sistemdeki frekans değişimi her iki kontrol sistemi için gösterilmiştir. Şekilden görüldüğü üzere, 0.03 pu'lik yük değişimi için, sistemin maksimum salınım (aşma) değeri bulanık PI kontrolörü için yaklaşık 0.02 pu iken klasik PI kontrolörü için 0.08 pu civarındadır. Ayrıca, sistem frekansının değişiminin bulanık PI kontrolörü için 9 sn içinde, klasik PI kontrolü için ise 13 sn içinde bastırıldığı görülmektedir.

Sisteme $\mathrm{t}=28$ sn anında -0.03 pu'lik yük değişimi uygulandığında, 0.03 pu'lik yük değişimine benzer olarak, sistemin maksimum salınım (aşma) değeri bulanık PI kontrolörü için yaklaş1k 0.02 pu iken klasik PI kontrolörü için 0.08 pu civarındadır. Ayrıca, sistem frekansının değişiminin bulanık PI kontrolörü için $\mathrm{t}=37$ sn anında, klasik PI kontrolü için ise $\mathrm{t}=41$ sn anında bastırıldığı görülmektedir. 


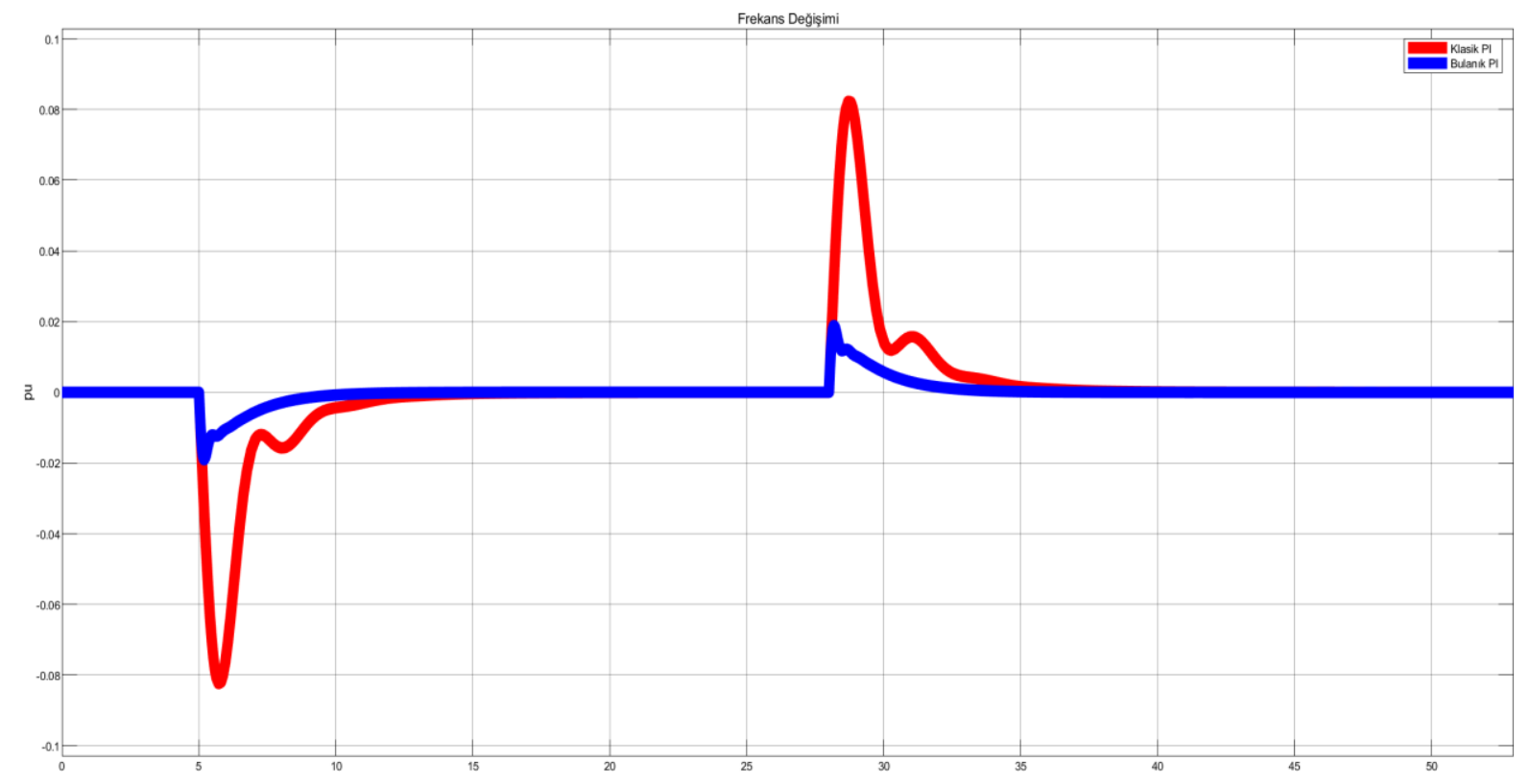

Şekil 10. 0.03 pu'lik ve -0.03 pu'lik yük değişimleri için frekans değişimi.

Şekil 11'de sisteme uygulanan 0.05 pu'lik ve -0.05 pu'lik yük değişimleri verilmiştir. Sisteme t=5 sn anında 0.05 pu'lik, t=28 sn anında -0.05 pu'lik yük değişimi uygulanmıştır.

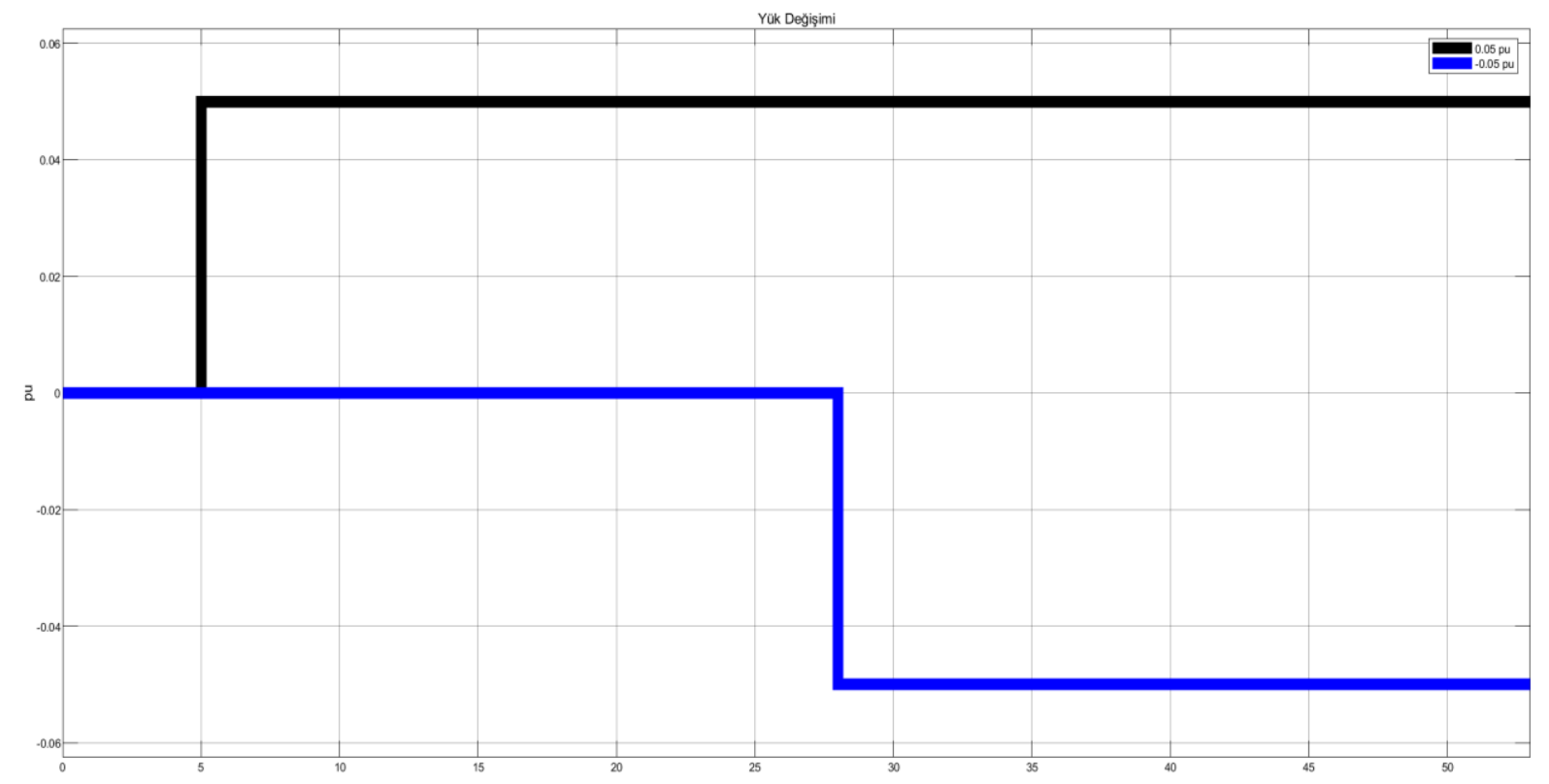

Şekil 11. $t=5$ sn için 0.05 pu'lik ve $t=28$ sn için -0.05 pu'lik yük değişimi.

Şekil 12'de ise bu yük değişimlerine karşı sistemdeki frekans değişimi her iki kontrol sistemi için gösterilmiştir. Şekilden görüldüğü üzere, 0.05 pu'lik yük değişimi için, sistemin maksimum salınım (aşma) değeri bulanık PI kontrolörü için yaklaşık 0.04 pu iken klasik PI kontrolörü için 0.14 pu civarındadır. Ayrıca, sistem frekansının değişiminin bulanık PI kontrolörü için 9 sn içinde, klasik PI kontrolü için ise 13 sn içinde bastırıldığı görülmektedir.

Sisteme $\mathrm{t}=28$ sn anında -0.05 pu'lik yük değişimi uygulandığında, 0.05 pu'lik yük değişimine benzer olarak, sistemin maksimum salınım (aşma) değeri bulanık PI kontrolörü için yaklaşık 0.04 pu iken 
klasik PI kontrolörü için 0.14 pu civarındadır. Ayrıca, sistem frekansının değişiminin bulanık PI kontrolörü için $\mathrm{t}=37$ sn anında, klasik PI kontrolü için ise $\mathrm{t}=41$ sn anında bastırıldığı görülmektedir.

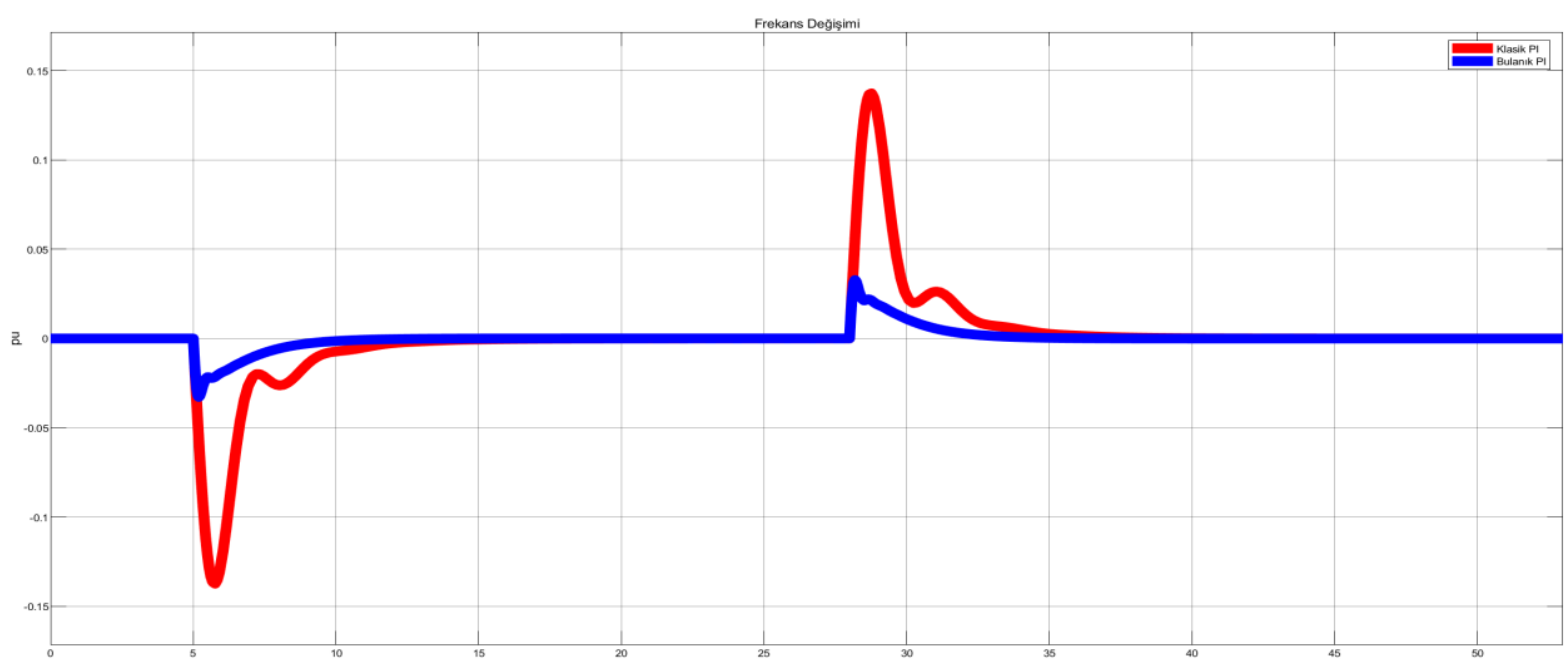

Şekil 12. 0.05 pu'lik ve -0.05 pu'lik yük değiş̧imleri için frekans değişimi.

\section{SONUC}

$\mathrm{Bu}$ çalışmada, tek bölgeli bir güç sisteminde YFK gerçekleştirilmiştir. Bunun için, öncelikle tek bölgeli güç sisteminin YFK modeli MATLAB/SIMULINK ortamında yapılmıştır. Daha sonra, kazancı bulanık mantıkla belirlenmiş bulanık PI ve klasik PI kontrolör yöntemleri kullanılarak systemin YFK işlemi gerçekleştirilmiştir. Her iki kontrolör için elde edilen sonuçlar karşılaştırılmıştır.

Yapılan simülasyonlar sonucunda, farklı değerdeki yük değişimleri için elde edilen değerler ve yapılan bazı çalışmalarda bulunan değerler Tablo 3'te verilmiştir. Tabloda verilen sonuçlardan, bulanık mantık kontrolör kullanılarak gerçekleştirilen YFK işleminde sistem frekansının 9 sn içinde nominal değerine getirildiği ve sistem frekansının istenen değerler $( \pm 200 \mathrm{mHz})$ arasında kalmasının sağlandığ görülmektedir.

Elde edilen sonuçlar benzer çalışmalarla kıyaslandığında, gerçekleştirilen çalışmada frekansın aşma değerinin daha küçük değerde olduğu görülmüştür. Ayrıca benzer çalışmalarda bulanık mantık kontrolörün farklı değerlerdeki yük değişimleri altındaki etkisi incelenmemiştir. Bu çalışmada daha farklı yük değişimleri ( \pm 0.03 pu ve $\pm 0.05 \mathrm{pu}$ ) uygulanarak sistem davranışı incelenmiştir. Elde edilen sonuçlar, uygulanan yöntemin farklı yük değişimleri için de iyi sonuçlar verdiğini göstermiştir. 
Tablo 3. Elde edilen sonuçlar.

\begin{tabular}{cccc}
\hline Yük Değişimi & Kontrolör Türü & $\begin{array}{c}\text { Oturma Zamanı } \\
(\mathrm{sn})\end{array}$ & $\begin{array}{c}\text { Aşma } \\
(\mathrm{pu})\end{array}$ \\
\hline & & 9 & 0.008 \\
$\pm 0.01 \mathrm{pu}$ & Bulanık Mantı & $32[1]$ & $3[1]$ \\
& & $6[2]$ & $0.0198[2]$ \\
$\pm 0.03 \mathrm{pu}$ & Bulanık Mantık & $10[9]$ & $0.0201[9]$ \\
\hline $0.05 \mathrm{pu}$ & Bulanık Mantık & $9.35[11]$ & $0.0145[11]$ \\
\hline
\end{tabular}

Sonuç olarak, bulanık mantık kontrol yönteminin daha iyi sönümleme, daha az aşma, daha az salınım ile daha iyi yanıtlar verdiği gösterilmiştir. Bu yüzden, daha kaliteli ve güvenilir bir elektrik enerji sağlanması noktasında, bulanık mantık kontrolörün yeterli olduğu söylenebilir.

\section{KAYNAKLAR}

[1] M. A. Zamee, D. Mitra and S. Y. Tahhan, "Load Frequency Control of Interconnected HydroThermal Power System Using Conventional PI and Fuzzy Logic Controller," International Journal of Energy and Power Engineering, vol. 2, no. 5, pp. 191-196, 2013.

[2] M. Karayel, E. Çelik ve İ. Yücedağ, "Bulanık Mantık Tabanlı Tek Bölgeli Yük Frekans Kontrolü," III. Uluslararası Bilimsel ve Mesleki Çalışmalar Kongresi-Mühendislik, 27-30 Haziran, Nevşehir/Türkiye, 2019.

[3] F. N. Saeed and A. J. Sultan, "Hybird PID-Fuzzy Controller for AGC in Two Thermal Area," Journal of Engineering and Applied Sciences, vol. 13, no. 21, pp. 9245-9251, 2018.

[4] S. Prakash and S. K. Sinha, "Load Frequency Control of Multi-Area Power Systems Using Neuro-Fuzzy Hybrid Intelligent Controllers," IETE Journal of Research, vol. 61, no. 5, pp. 526-532, 2015.

[5] K. Choudhary and B. Singh, "Load Frequency Control in Two Area Power Systems Integrated with SMES Combination Using Fuzzy-PID and ANFIS Controller," International Research Journal of Engineering and Technology (IRJET), vol. 6, no. 6, pp. 2335-2341.

[6] E. Çam and İ. Kocaarslan, "Load Frequency Control in Two Area Power Systems Using Fuzzy Logic Controller," Energy Conversion and Management, vol. 46, pp. 233-243, 2005. 
[7] E. Özkop, İ. H. Altaş ve A. S. Akpınar, "Bulanık Mantık Denetleyicili Güç Sistem Uygulamas1," Elektrik-Elektronik ve Bilgisayar Mühendisliği Sempozyumu (ELECO), Aralık, Bursa/Türkiye, 2004.

[8] M. R. Tur, M. Wadi, A. Shobole and S. Ay, "Load Frequency Control of Two Area Interconnected Power System Using Fuzzy Logic Control and PID Controller," 7th International Conference on Renewable Energy Research and Applications, 14-17 October, Paris/France, 2018.

[9] E. Çam ve İ. Kocaarslan, "Tek Bölgeli Güç Sistemlerinde Bulanık Mantık ile Yük Frekans Kontrolü,” Teknoloji, c. 3, s. 4, ss. 73-77, 2002.

[10] A. Kumar, P. Rai and Dr. Ghanshyam, "Automatic Generation Control: - A Fuzzy Logic Approach," International Journal of Engineering Research \& Technology, vol. 2, no. 10, 2013.

[11] A. Karyeyen, N. Çetinkaya ve S. Güntay, "İki Bölgeli Elektrik Güç Sistemlerinde Optimum Yük Frekans Kontrolü,” Elektrik-Elektronik-Bilgisayar ve Biyomedikal Mühendisliği 13. Ulusal Kongresi ve Fuarında sunuldu, 2009.

[12] S. J. Safi, S. S. Tezcan, İ. Eke and Z. Farhad, "Gravitational Search Algorithm (GSA) Based PID Controller Design for Two Area Multi-Source Power System Load Frequency Control (LFC)," Gazi University Journal of Science, vol. 31, no. 1, pp. 139-153, 2018.

[13] S. Duman, N. Yörükeren and İ. H. Altaş, "Load Frequency Control of A Single Area Power System Using Gravitational Search Algorithm," International Symposium on Innovations in Intelligent System and Applicatioms, Trabzon/Turkey, 2012.

[14] P. Mohanty, R. K. Sahu and S. Panda, "A Novel Hybrid Many Optimizing Liaisons Gravitational Search Algorithm Approach for AGC of Power Systems," Automatika Journal for Control, Measurement, Electronics, Computing and Communications, vol. 61, no. 1, pp. 158-178, 2020.

[15] S. Duman and N. Yörükeren, "Automatic Generation Control of the Two Area Non-Reheat Thermal Power System Using Gravitational Search Algorithm," Przeglad Elektrotechniczny (Electrical Review), 2012.

[16] K. Alzaarer, A. Q. Al-Shetwi, C. Z. El-Bayeh and M. B. Taha, "Automatic Generation Control of Multi-Area Interconnected Power Systems Using ANN Controller," International Information and Engineering Technology Association, vol. 34, no. 1, pp. 1-10, 2020.

[17] S. Prakash and S. K. Sinha, "Application of Artificial Intelligence in Load Frequency Control of Interconnected Power System," International Journal of Engineering, Science and Technology, vol. 3, no. 4, pp. 264-275, 2011.

[18] A. Demirören, H. L. Zeynelgil and N. S. Şengör, "Automatic Generation Control Using ANN Technique for Multi-Area Power System with SMES Units," Electric Power Components and Systems, vol. 32, pp. 193-213, 2004. 
[19] A. Sharma, K. P. S. Parmar and S. K. Gupta, "Automatic Generation Control Using ANN," National Conference on Recent Advancements in Science, Technology and Management, November, Indore/India, 2011.

[20] M. Krishnan, "Automatic Generation Control of Multi Area Power System Using Fuzzy Logic and ANN Controller,” RUAS-SASTech Journal, vol. 16, no. 1, 2017.

[21] M. T. Özdemir ve D. Öztürk, "İki Bölgeli Güç Sisteminin Optikten Esinlenen Optimizasyon Algoritması ile Optimal Yük Frekans Kontrolü," Firat Üniversitesi Mühendislik Bilimleri Dergisi, c. 28, s. 2, ss. 57-66, 2016.

[22] B. Dhanasekaran and S. Siddhan, "Implementation of Load Frequency Control for Single Area Multi Source Power System," International Conference in Green, Intelligent Computing\&Communiciation Systems, April, Coimbaore/India, 2020.

[23] H. Saraçoğlu and A. Demirören, "AGC with Fuzzy Controller Parameters are Tuned By GA," 6th International Conference on Electrical Engineering (ICEENG), 27-29 May, Cairo/Egypt, 2008.

[24] E. Yalçın, E. Çam, T. Vardar ve M. Lüy, "PID Kontrolör ile İki Bölgeli Güç Sistemlerinde Yük Frekans Kontrolünün İncelenmesi,” International Journal of Engineering Research and Development, vol. 5, no. 2, 2013.

[25] G. El-Saady, El-Nobi A. İbrahim and A. H. Okilly, "HVDC FACTS Controller for Load Frequency Control System," Fourth International Conference on Energy Engineering (ICEE-4), 26-28 December, Aswan/Egypt, 2017.

[26] M. I. Alomoush, "Load Frequency Control and Automatic Generation Control Using Fractional-Order Controllers," Electrical Engineering, vol. 91, pp. 357-368, 2010. 